\title{
Membrane protein CD9 is repositioned and released to enhance uterine function
}

\author{
Maki Iwai ${ }^{1,2} \cdot$ Toshio Hamatani $^{1} \cdot$ Akihiro Nakamura $^{1,2} \cdot$ Natsuko Kawano $^{2,3} \cdot$ Seiya Kanai ${ }^{3} \cdot$ Woojin Kang $^{2,4}$. \\ Noriko Yoshii ${ }^{5} \cdot$ Yasushi Odawara ${ }^{6} \cdot$ Mitsutoshi Yamada $^{1} \cdot$ Yoshitaka Miyamoto $^{2} \cdot$ Takakazu Saito $^{4}$. \\ Hidekazu Saito ${ }^{4} \cdot$ Mami Miyado $^{7} \cdot$ Akihiro Umezawa $^{2} \cdot$ Kenji Miyado $^{2} \cdot$ Mamoru Tanaka $^{1}$
}

Received: 11 July 2018 / Revised: 6 August 2018 / Accepted: 21 August 2018 / Published online: 6 November 2018

(c) United States \& Canadian Academy of Pathology 2018

\begin{abstract}
Tetraspanin CD9 is essential for sperm-egg fusion and also contributes to uterine repair through microexosome formation. Microexosomes share CD9 with exosomes and are released from eggs and uterine epithelial cells. However, the mechanism for the formation of microexosomes remains unknown. To address this issue, we examined membrane localization and extracellular release of CD9 proteins using uterine epithelial cells and secretions in mice and humans. In mice, CD9 localized predominantly on the basal region of the plasma membrane and relocated to the apical region upon embryo implantation. Furthermore, extracellular CD9 proteins were detected in uterine secretions of mice and women undergoing infertility treatment, but were below detectable levels in supernatants of pluripotent stem cells. Ultrastructural analysis demonstrated that membrane projections were shortened and the number of mitochondria was reduced in uterine epithelial cells lacking $C d 9$ genes. Our results suggest that $\mathrm{CD} 9$ repositioning and release affect both membrane structures and mitochondrial state in the uterus, and contribute to female fertility.
\end{abstract}

\section{Introduction}

In humans, uterine repair after normal menstruation and childbirth has features in common with tissue repair in other organs [1-3]. During uterine repair, the human endometrium undergoes the menstrual cycle [1], whereas other mammals are subject to the estrous cycle [4]. In both humans and mice,

Electronic supplementary material The online version of this article (https://doi.org/10.1038/s41374-018-0145-1) contains supplementary material, which is available to authorized users.

Toshio Hamatani

toshiohamatani@z3.keio.jp

$\triangle$ Kenji Miyado

miyado-k@ncchd.go.jp

1 Department of Obstetrics and Gynecology, Keio University School of Medicine, 35 Shinanomachi, Shinjuku, Tokyo 1608582, Japan

2 Department of Reproductive Biology, National Research Institute for Child Health and Development, 2-10-1 Okura, Setagaya, Tokyo 157-8535, Japan

3 Department of Life Sciences, School of Agriculture, Meiji the endometrium commonly grows to a thick and blood vessel-rich glandular layer, providing the optimal environment for embryo implantation on the uterine surface. The endometrium consists of the columnar epithelium and connective tissues that vary in thickness in response to hormonal regulation. It undergoes extensive epithelial turnover throughout adult female life. In mice, the estrous cycle is divided into two ovarian phases, the follicular and the luteal phases [4]. The follicular phase is the period of ovarian follicle development consisting of proestrus and estrus; the luteal phase is the period of corpus luteum formation, comprising

University, 1-1-1 Higashimita, Kawasaki, Kanagawa 214-8571, Japan

4 Department of Perinatal Medicine and Maternal Care, National Center for Child Health and Development, 2-10-1 Okura, Setagaya, Tokyo 157-8535, Japan

5 Tokyo Adventist Hospital Megumi Clinic, 3-5-2 Amanuma, Suginami, Tokyo 167-0032, Japan

6 Fertility Clinic Tokyo, 3-13-11 Higashi, Shibuya, Tokyo 1500011, Japan

7 Department of Molecular Endocrinology, National Research Institute for Child Health and Development, 2-10-1 Okura, Setagaya, Tokyo 157-8535, Japan 
metestrus and diestrus. These four stages are easily distinguished using a vaginal smear test (Fig. 1a).

The uterus is a hormone-responsive muscular organ that is responsible for embryonic development [5, 6]. It is a distensible organ that expands during pregnancy, becoming large enough to hold a fully grown baby. The physical and oxidative stresses of pregnancy and childbirth frequently damage uterine and placental tissues, especially in women who experience multiple childbirths [7, 8]. Uterine repair involves a number of cellular processes, including hypertrophy and rearrangement of uterine tissues, and changes in the extracellular matrix $[9,10]$.

CD9 is an integral membrane protein expressed in a wide variety of cells $[11,12]$. In human and mouse uteri, CD9 is predominantly localized on the plasma membrane of endometrial epithelial cells $[13,14]$. In addition, CD9-containing extracellular structures, exosomes [15], and microexosomes $[14,16-18]$, are released from cells to the outer environment in many different types of cells. Specifically, microexosomes are extracellular structures without typical bilayers [16-18], predicted to function in the short-range transportation of cellular substances between neighboring cells [18]. In eggs, microexosomes are released from the plasma membrane and transferred to the sperm head, promoting the fusion of egg with sperm [16]. In the uterus, microexosomes are released from epithelial cells, repairing the epithelium damaged by childbirth [14]. However, it is unknown how microexosomes are formed in the uterus. To address this issue, we focused on CD9 localization on the plasma membrane of the uterine epithelium.

\section{Materials and methods}

\section{Animal experiments}

All mice were housed under specific pathogen-free conditions. Food and water were available ad libitum. All animal a

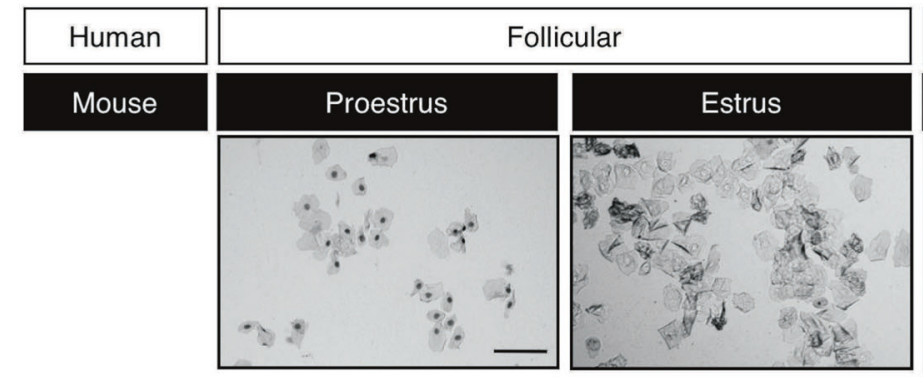

b
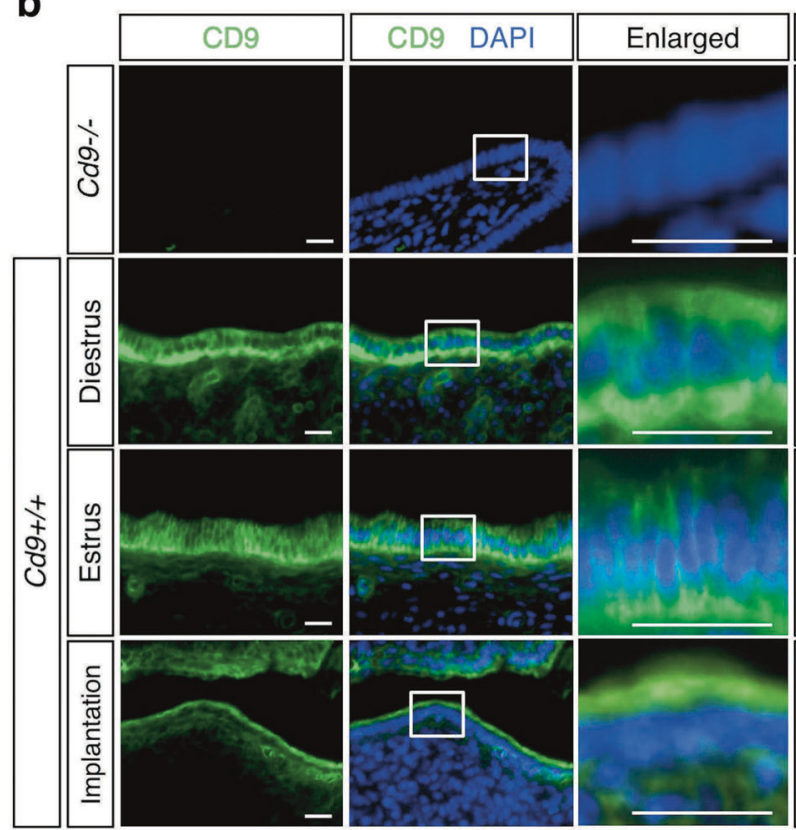

Fig. 1 CD9 localization during estrous cycle and at implantation. a Staging of the estrous cycle by vaginal cytology; scale bar, $50 \mu \mathrm{m}$. b Immunostaining of uterine tissues with anti-CD9 mAb; chromosomal DNA was counterstained with DAPI; uteri were staged at diestrus and estrus, and during implantation were sectioned; scale bar, $20 \mu \mathrm{m}$. c
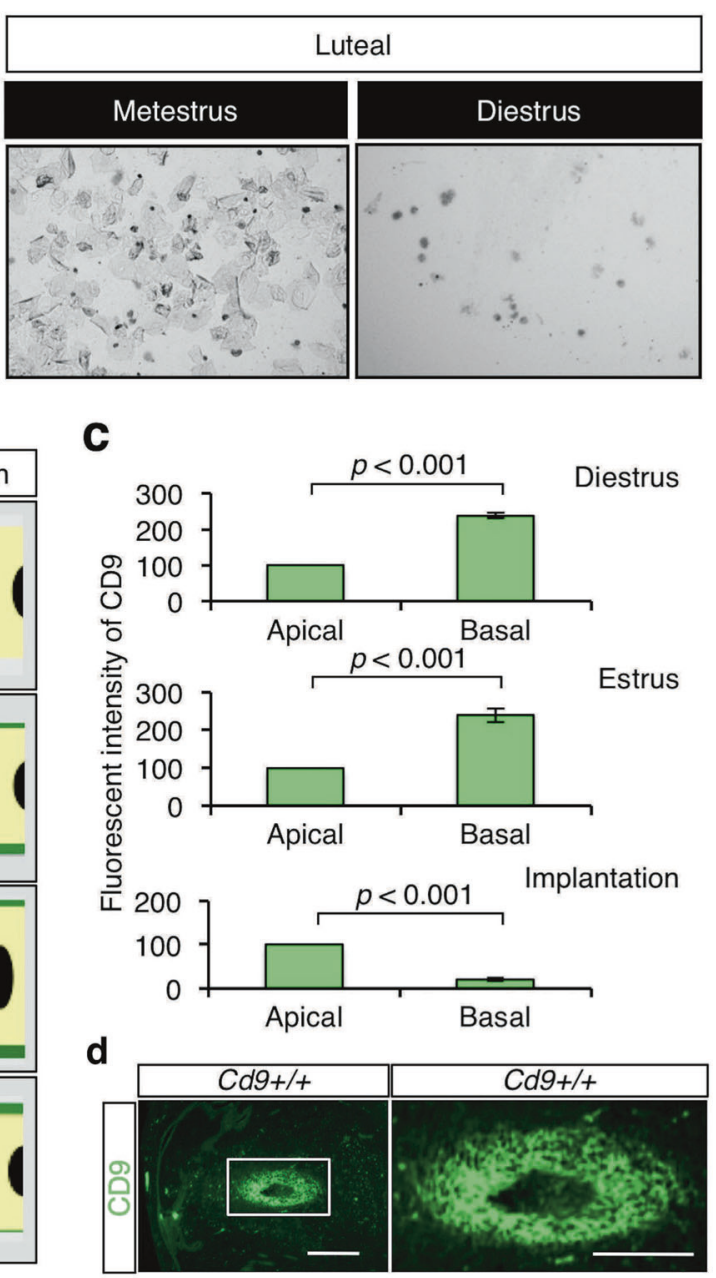

Relative fluorescence intensity of uterine epithelial cells immunostained with anti-CD9 between apical and basal regions; intensity of the apical region was set to 100 ; values were expressed as mean $\pm \mathrm{SE}$. d Immunostaining of the site of embryo implantation with anti-CD9 $\mathrm{mAb}$; scale bar, $100 \mu \mathrm{m}$ 
experiments were performed in accordance with the principles and guidelines of the Care and Use of Laboratory Animals at the National Research Institute for Child Health and Development. The animal committee of the National Research Institute for Child Health and Development approved all experiments, including animal experiments (experiment number 04-004).

\section{Human cell culture}

Menstrual blood cell lines (Edom22) were established in our laboratory [19]. Edom22 cells were maintained in POWEREDBY10 medium (MED SHIROTORI CO., Ltd, Tokyo, Japan). Human induced pluripotent stem cells (hiPSCs) were generated in our laboratory, via procedures described by Yamanaka and colleagues [20], with slight modifications [21]. The human cells were infected with retroviruses produced from the retroviral vector pMX, which carries complementary DNAs that encode human OCT3/4, SOX2, cMYC, and KLF4. Human iPSCs were cultured on irradiated mouse embryonic fibroblasts in 0222 medium supplemented with $10 \mathrm{ng} / \mathrm{mL}$ recombinant human basic fibroblast growth factor (Wako Pure Chemical Industries, Ltd., Osaka, Japan).

\section{Animals}

Eight- to 12-week-old female C57BL/6 mice were purchased from SLC (Shizuoka, Japan). $C d 9^{-/-}$(Cd9 knockout) mice were generated as described previously [22] and backcrossed with a C57BL/6 genetic background. $C d 9^{-1-}$ mice expressing $\mathrm{CD} 9$ tagged at the $\mathrm{N}$-terminus with enhanced green fluorescent protein (CD9-eGFP) $\left(C d 9^{-/} T G\right)$ were generated as described previously [16]. The genotypes of mice were determined by standard procedures as described previously $[14,16]$.

\section{Antibodies and chemicals}

For immunohistochemistry and immunoblotting, antimouse CD9 (KMC8) and anti-human CD9 (ALB6) monoclonal antibodies (mAbs) were purchased from BD Biosciences (San Jose, CA), and an anti-vascular endothelial growth factor (VEGF) (Ab4) polyclonal antibody (polyAb) was purchased from Merck KGaA (Darmstadt, Germany). Alexa Fluor 488- and 546-conjugated secondary Abs (Invitrogen/Thermo Fisher Scientific, USA) were used for immunohistochemistry. Horseradish peroxidase (HRP)conjugated secondary antibodies (Abs) (Sigma-Aldrich) were used for immunoblotting. Nuclei were counterstained with 4', 6-diamidino-2-phenylindole (DAPI) (WAKO Pure Chemical Industries).
Vaginal smear test and collection of mouse uterine secretions

Wild-type $\left(C d 9^{+/+}\right)$and $C d 9^{-/} T G$ female mice were examined by vaginal smear cytology and assigned to one of four phases of the estrous cycle as described previously [14]. Briefly, a vaginal smear was collected with a moistened cotton swab, applied to a glass slide, stained with hematoxylin, and then observed under a stereomicroscope. According to the microscopic characteristics of the vaginal smear, mice were classified as being in proestrus, estrus, metestrus, or diestrus. Because the uterus undergoes hormonal changes during the estrous cycle and is distended at the estrus stage because of increased uterine secretions, we collected the uterine secretions from estrus-staged mice. Mice staged at estrus were sacrificed and their uteri were incised and flushed with $50 \mu \mathrm{L}$ solution, phosphate-buffered saline (PBS) and $1 \%$ Triton X-100 solution. The fluids collected with the PBS wash contained the uterine secretions and those collected with the Triton X-100 solution contained components derived from epithelial cells, such as membrane and cytoplasmic proteins. After these solutions used to flush the uteri were collected from each of the uterine horns, they were subjected to immunoblotting as described below.

\section{Collection of human uterine secretions}

Patients were recruited from the outpatient department of the Fertility Clinic Tokyo and St. Women's Clinic, Tokyo, and the Department of Obstetrics and Gynecology, Keio University School of Medicine, Japan. They were over 43 years of age and provided informed consent to participate. This study was approved by the ethics committee at the Japanese Institution for Standardizing Assisted Reproductive Technology (\#11-09). Samples were collected only from patients who had provided informed consent.

Patient samples were collected during the mid-luteal phase at around day 20 of their contraceptive cycles, which is considered an implantation window. A catheter connected to a disposable 5-mL syringe (Terumo, Japan) was inserted into the uterine cavity through the cervix. Five milliliters of saline (Otsuka Pharmaceutical) was then injected into the cavity, aspirated immediately, and collected in Eppendorf tubes. The collected uterine secretions were centrifuged at $1500 \mathrm{rpm}$ for $10 \mathrm{~min}$ at room temperature to remove blood corpuscles and large protein complexes. The supernatants were then transferred to new Eppendorf tubes. The protein concentration of each sample was measured by the biuret test. 


\section{Immunoblotting of uterine secretions}

Immunoblotting was performed as described previously [14]. Briefly, 50- $\mu \mathrm{L}$ samples of human or mouse uterine secretions were boiled at $95{ }^{\circ} \mathrm{C}$ for $10 \mathrm{~min}$ in $50 \mu \mathrm{L}$ of Laemmli's sodium dodecyl sulfate (SDS) sample buffer containing 2\% SDS, $62.5 \mathrm{mM}$ Tris- $\mathrm{HCl}$ (pH 6.8), $0.005 \%$ bromophenol blue, and $7 \%$ glycerol. Then, $20 \mu \mathrm{L}$ of each sample was resolved by SDS-polyacrylamide gel electrophoresis on $10 \%$ acrylamide gels, then transferred to a polyvinylidene difluoride (PVDF) membrane (Immobilon$\mathrm{P}$, Millipore). The Abs of interest were bound to proteins on the PVDF membranes and detected with chemiluminescence using HRP-conjugated secondary immunoglobulin G Abs (IgGs) and an ECL Western Blotting System substrate (GE Healthcare, UK Ltd). Band intensities were measured using ImageJ software (NIH).

\section{Immunoblotting of supernatants of pluripotent stem cells}

As reported previously [23], mouse embryonic stem cells (mESCs) were established from blastocysts developed from fertilized eggs using mouse ESC-derivation medium, knockout Dulbecco's modified Eagle's medium (Life Technologies, CA, USA), supplemented with $15 \%$ knockout serum replacement, $1 \mathrm{X}$ non-essential amino acids, 0.05 $\mathrm{mM}$ 2-mercaptoethanol, $2 \mathrm{mM}$ GlutaMAX, $100 \mathrm{U} / \mathrm{mL}$ penicillin $\mathrm{G}, 10 \mathrm{mg} / \mathrm{mL}$ streptomycin (all from Life Technologies), $1000 \mathrm{U} / \mathrm{mL}$ leukemia inhibitory factor (LIF) (Wako, Japan), and the MEK kinase inhibitor PD98059 (Cell Signaling Technology, Danvers, MA). For serum-free and feeder-free cultures, mESCs were cultured in $\mathrm{N}-2$ and B-27 medium (Life Technologies), supplemented with $1000 \mathrm{U} / \mathrm{mL}$ LIF, $3 \mu \mathrm{M}$ CHIR99021 (Wako), and $1 \mu \mathrm{M}$ PD0325901 (Wako) on Matrigel-coated dishes (BD Biosciences, San Jose, CA). Cultured media of mESCs and hiPSCs were collected and centrifuged at 10,000 rpm at $4{ }^{\circ} \mathrm{C}$ for $5 \mathrm{~min}$. The supernatants were divided into two parts, upper and lower, which were subjected to immunoblotting.

\section{Gel filtration chromatography}

Uterine secretions collected from mice and women was separated by gel filtration using Econo-Column Chromatography Columns $(1.0 \times 20 \mathrm{~cm} \quad \# 7371022) \quad$ (Bio-Rad Laboratories, Inc., Hercules, CA) and Sephacryl S-1000 Superfine gel filtration resin (GE Healthcare, UK Ltd) as described previously [24]. After each purification step, fractions containing CD9 proteins were analyzed by immunoblotting. Band intensities were measured using ImageJ software (NIH).
Immunohistochemical analysis of mouse uterine tissues

Estrus-staged female mice were sacrificed, and their uteri were excised and fixed with $4 \%$ paraformaldehyde for $3 \mathrm{~h}$ at $4{ }^{\circ} \mathrm{C}$. After fixation, the uterine tissues were immersed in a $30 \%$ sucrose solution at $4{ }^{\circ} \mathrm{C}$ until they sank to the bottom of the conical tubes; they were then embedded in OCT (Tissue-Tek) and frozen at $-80^{\circ} \mathrm{C}$. Subsequently, the tissues were sectioned at $10 \mu \mathrm{m}$ with a cryostat (CryoStar NX70, Thermo Scientific). The sections were dried and incubated with primary Abs $(2.5 \mu \mathrm{g} / \mathrm{mL})$ in HEPES-buffered saline [HBS: $10 \mathrm{mM}$ HEPES (pH 8.0), $0.15 \mathrm{M} \mathrm{NaCl}$, and $3 \%$ fetal bovine serum (FBS)] for $2 \mathrm{~h}$ at room temperature, and then incubated with Alexa Fluor 488- or 546-conjugated IgGs, followed by three washes in HBS. Nuclei were then counterstained with DAPI at a final concentration of $10 \mu \mathrm{g} / \mathrm{mL}$ in HBS for $30 \mathrm{~min}$ at room temperature, followed by three washes in HBS. Images were captured under a laser scanning confocal microscope (LSM 510; Carl Zeiss MicroImaging, Inc., Thornwood, NY).

\section{Immunoelectron and electron microscopic analyses}

Uterine secretions collected from $C d 9^{+/+}$and $C d 9^{-/} T G$ female mice were fixed with glutaraldehyde and osmic acid solutions. Ultrathin sections were prepared as described previously [25]. To compare the number of microvilli and mitochondria between $C d 9^{+/+}$and $C d 9^{-/} T G$ female mice, we counted microvilli on the plasma membrane and mitochondria in the cytoplasm of ten epithelial cells.

\section{Statistical analysis}

Comparisons were made using one-way analysis of variance (ANOVA) followed by a post-hoc Scheffe test (Scheffe's method), the Mann-Whitney $U$-test, or Fisher's exact test. Statistical significance was defined as $p<0.05$. Results were expressed as the mean \pm standard error (SE).

\section{Results}

\section{CD9 localization in uterine epithelial cells}

To explore a mechanism of microexosome formation, we observed membrane localization of CD9 proteins in uterine epithelial cells during the estrous cycle (Figs. 1b, c). The uterine epithelial cells of wild-type $\left(C d 9^{+/+}\right)$female mice were immunostained with anti-mouse CD9 mAb. Concomitantly, the uterine epithelial cells of $C d 9^{-/-}$female mice were treated as a negative control. At the diestrus stage, $\mathrm{CD} 9$ was localized at the basal region rather than the apical 
region. The fluorescence intensity of the basal region was higher than that of the apical region $(238.5 \pm 24.8$ vs. 100.0, respectively; $n=20 ; p<0.001)$. Similarly, CD9 was localized at the basal region rather than the apical region at the estrus stage $(238.7 \pm 58.3$ vs. 100.0 , respectively; $n=20$; $p<0.001)$. In contrast, CD9 localization reversed at the implantation stage and its intensity was higher at the apical region than the basal region (100.0 vs. $20.0 \pm 10.1$, respectively; $n=20 ; p<0.001)$. More specifically, CD9 proteins accumulated at the region where embryos implanted (Fig. 1d). From these results, we inferred that CD9 localization is subject to repositioning on the plasma membrane of uterine epithelial cells.

\section{CD9 proteins in uterine secretions}

To examine the universality of the extracellular release of CD9 proteins, we detected CD9 proteins in the supernatants of distinct types of cells. The uterine secretions were subjected to immunoblotting (Fig. 2a and Supplemental Fig. 1a). CD9 was detected in all six mice examined. In turn, female mice were divided into four stages according to a vaginal smear check (Fig. 1a). The predominant feature of proestrus stage is the presence of small, round, nucleated epithelial cells with no neutrophils. Estrus stage is characterized by the presence of predominantly anucleated keratinized epithelial cells. Metestrus stage is characterized by a a
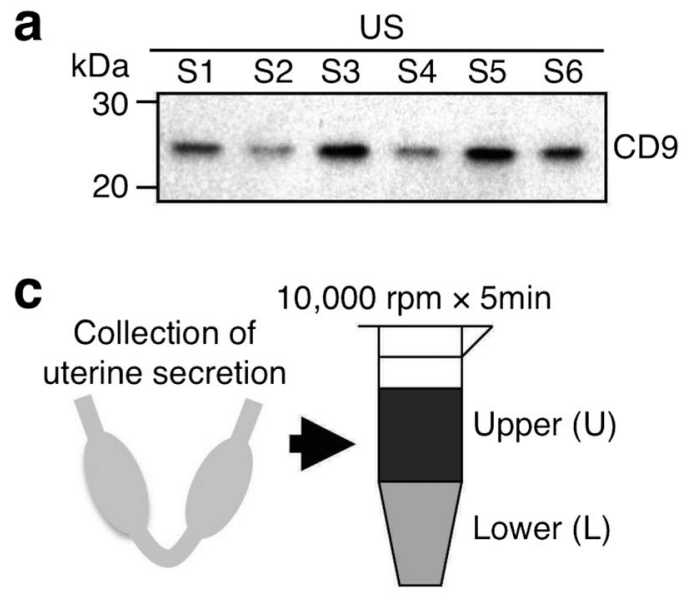

d

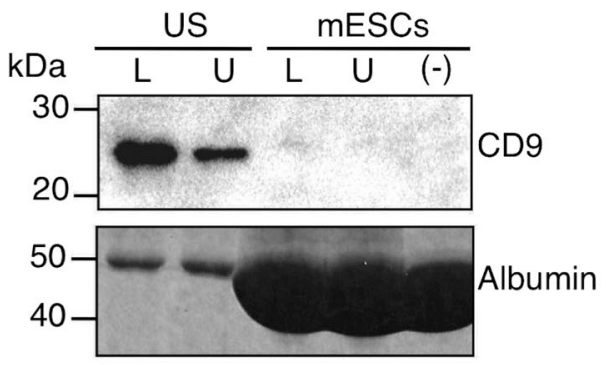

e

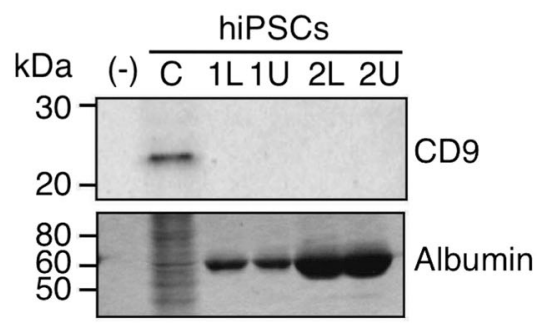

b
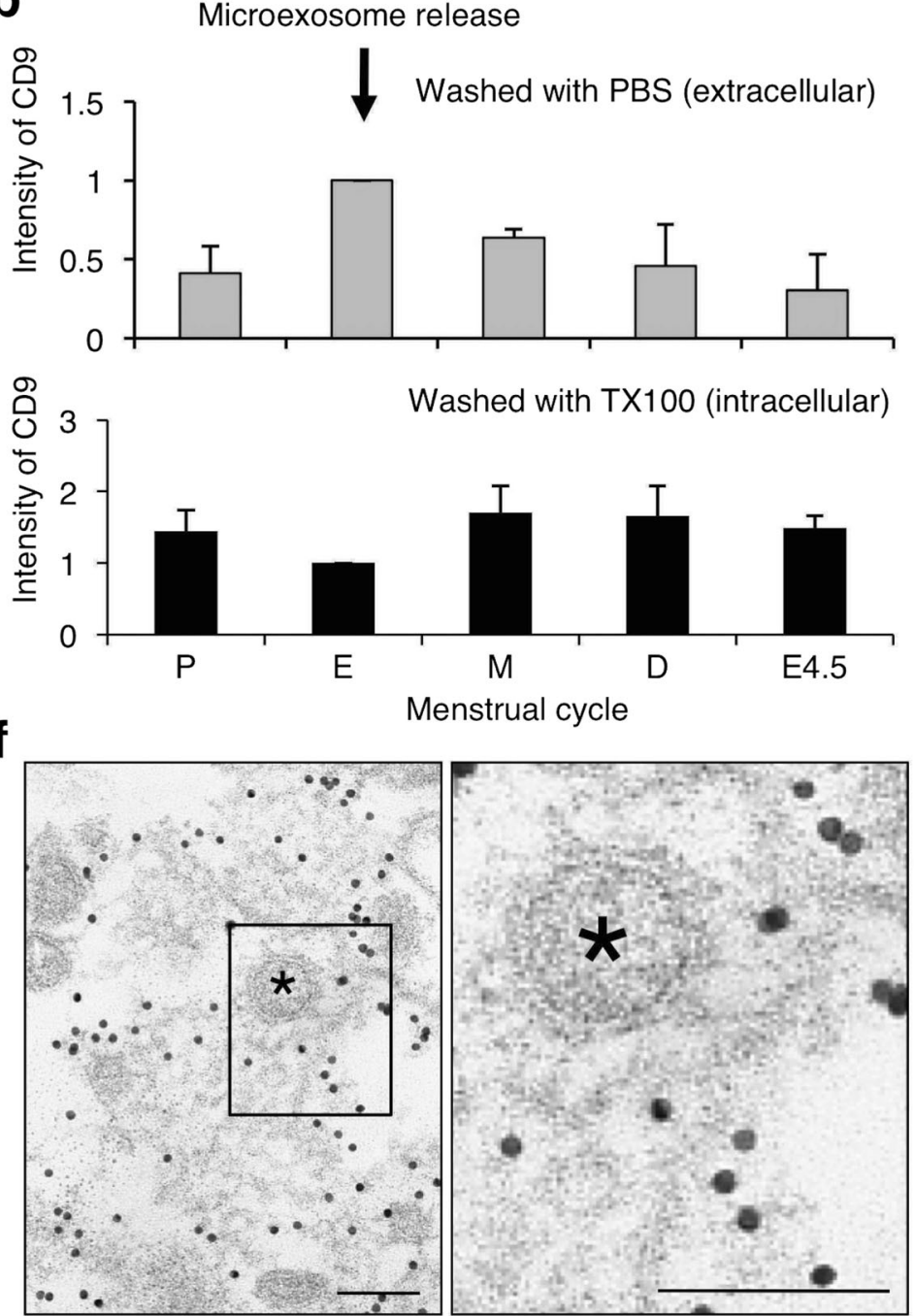

Fig. 2 Immunoblotting of uterine secretions and supernatants of cultured stem cells with anti-CD9 mAb. a Mouse uterine secretions (6 samples, S1-S6). b Mouse uterine secretions staged by vaginal smear test; uterine secretions were collected with PBS and then washed with Triton X-100 solution; P, proestrus; E, estrus; M, metestrus; D, diestrus; E4.5, implantation stage. c Estrus-staged mouse uterine secretions and mouse embryonic stem cells (mESCs) after centrifugation $(10,000 \mathrm{~g}$ for $5 \mathrm{~min}$ ); after centrifugation, samples were divided into lower (L) and upper (U) parts. d Mouse uterine secretions and mESCs after centrifugation; L, lower parts; U, upper parts; as an internal control, albumin was stained with Coomassie Brilliant Blue (CBB) solution. e HiPSCs after centrifugation (two independent samples); C, cellular extract; L, lower parts; U, upper parts. f Immunoelectron microscopic analysis of mouse uterine secretions staged at estrus with anti-CD9 mAb; Asterisk indicate exosome with typical lipid bilayers; scale bar, $100 \mathrm{~nm}$ 
combination of anucleated keratinized epithelial cells, neutrophils, and nucleated epithelial cells. Diestrus stage is characterized by the presence of predominantly neutrophils. Uterine secretions were collected from the uterine cavity of two groups, one group without implanted embryos and one group with implanted embryos at embryonic day 4.5 (E4.5) (Fig. 2b). The uterine secretions were subjected to immunoblotting and the band intensities of CD9 proteins were estimated densitometrically and compared with a value of 1.0 in estrus-staged mice (Fig. 2b). As reported previously [14], CD9 was the most abundant in the uterine secretions collected from estrus-staged mice including group with implanted E4.5 (Fig. 2b). In contrast, CD9 was the scarcest in the epithelial cells of estrus-staged mice (Fig. 2b). These results suggest that CD9 is released from estrus-staged epithelial cells and stored in the uterine cavity throughout all stages.

Although centrifugation was performed (Fig. 2c), CD9 was detected in both the upper (U) and lower (L) supernatants in estrus-staged uterine secretions (Fig. 2d and Supplemental Fig. 1b). In contrast, CD9 was undetectable in mESCs in both $\mathrm{U}$ and L supernatants (Fig. 2d and Supplemental Fig. 1b). Even when immunoblotting was carried out independently for two samples of hiPSCs derived from menstrual blood cell lines (Edom22), CD9 was detected in the cells but not the

a

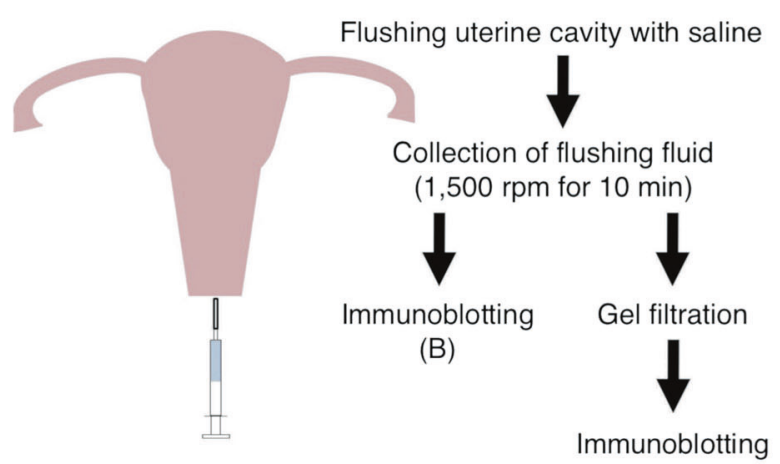

(C)

\section{b}

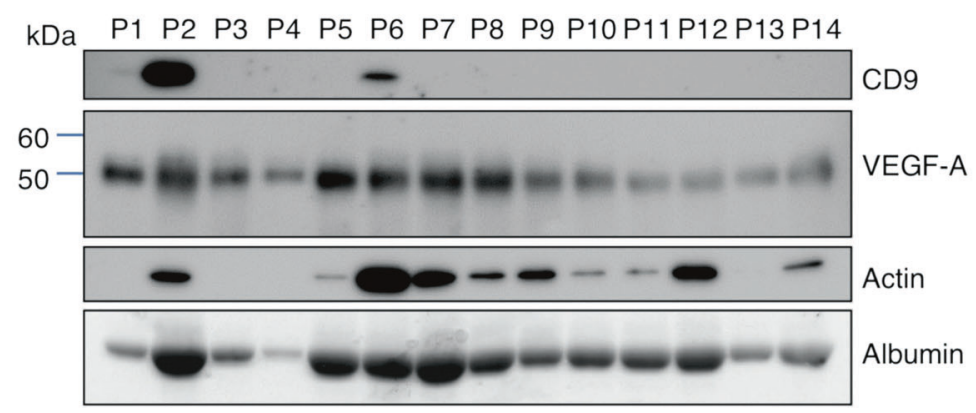

supernatants (Fig. 2e and Supplemental Fig. 2). To investigate the structural features of $\mathrm{CD} 9$-containing vesicles, we carried out immunoelectron microscopic analysis of uterine secretions collected from estrus-staged mice (Fig. 2f). The structures that immunoreacted with anti-CD9 mAb conjugated with gold particles $(10 \mathrm{~nm})$ had no typical lipid bilayers, indicating that CD9-containing structures are microexosomes, but not exosomes. This result suggests that CD9-containing microexosomes are stored in the uterine secretions of estrusstaged mice in a manner specific to uterine tissue.

\section{Microexosomes in human uterine secretions}

To examine the presence of microexosomes in human uterine secretions, uterine secretions were collected from women (Fig. 3a, Supplemental Fig. 3 and 4) and subjected to immunoblotting (Fig. 3b). As in mouse uterine secretions, CD9 was detected in human uterine secretions, but CD9-positive samples were limited. When the patients were separated into those with thin endometrium at the mid-luteal phase ( $<8.5 \mathrm{~mm}$ by transvaginal ultrasound) or those with normal-width endometrium ( $\geqq 8.5 \mathrm{~mm})$, the CD9-negative rate was significantly higher in the patients with thin endometrium $(81.8 \%, n=22)$ than those with normal
Fig. 3 Gel filtration chromatography of human uterine secretions. a Experimental flow; human uterine secretions were collected by flushing uterine cavity with saline and examined by immunoblotting (b) and gel filtration chromatography (c). b Immunoblotting of human
C
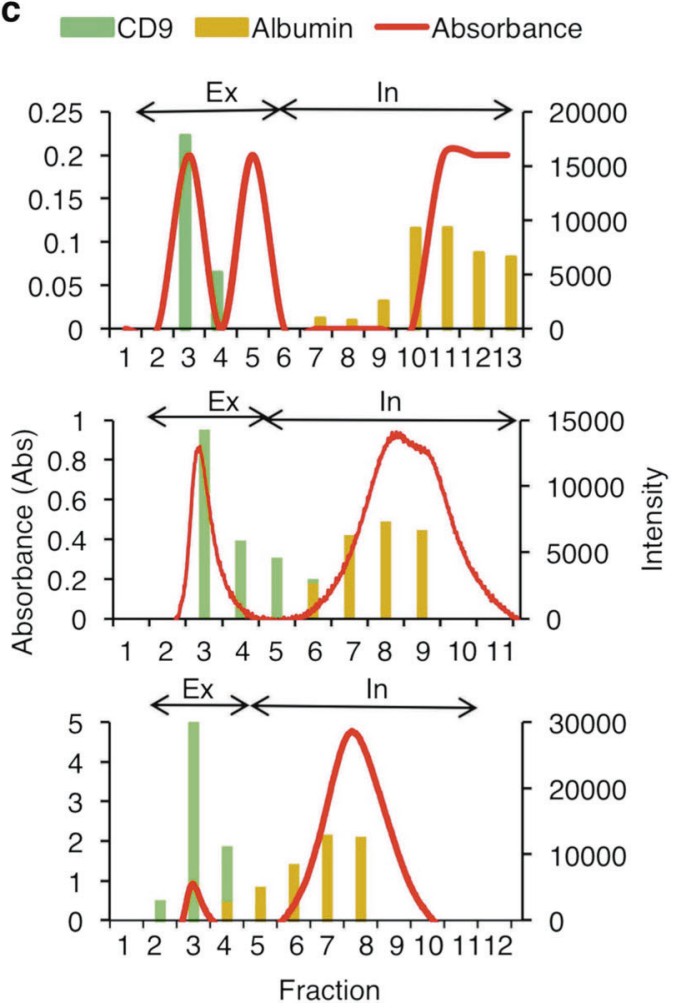

uterine secretions with anti-CD9, VEGF-A, and actin mAb; albumin was stained with $\mathrm{CBB}$ solution. $\mathbf{c}$ Gel filtration chromatography of human exclusion factor (Ex) and inclusion factor (In) 
endometrium $(49.3 \%, n=69)$. As reported previously [14], the amount of CD9 protein present in the samples was correlated to endometrial thickness, which is a potent prognostic factor for infertility [26, 27]. Furthermore, VEGF is a secretory protein that promotes the growth of new blood vessels [26]. It activates the mammalian target of rapamycin (mTOR) signaling pathway to upregulate mitochondrial function [27], and rapamycin inhibits cell proliferation [28]. In addition, uterine epithelial cells release VEGF-A during microexosome formation [14]. As VEGFA was detected in the samples from all women (Fig. 3b), epithelial VEGF-A proteins may be indistinguishable from VEGF-A proteins derived from blood.

CD9-positive samples presumably possess the normal characteristics of human uterine secretions. Therefore, we estimated the size of uterine microexosomes using gel filtration chromatography. When three samples expressing CD9 were examined, most of the CD9 proteins, as well as the bulk of all other proteins, were recovered in the exclusion fraction (Ex) at void volume (Vo) and in the inclusion fraction (In) at total volume (Vt), respectively (Fig. 3c). This result indicates that the larger structures containing CD9, presumably linked to microexosomes, are contained in human uterine secretions.

\section{Ultrastructural features of estrous-staged epithelial cells}

To examine the features of cells releasing microexosomes, we performed electron microscopic analysis of uterine epithelial cells in $C d 9^{+/+}$and $C d 9^{-/-}$mice expressing CD9 in eggs $\left(C d 9^{-/} T G\right)$. These mice produce a first litter of pups, a

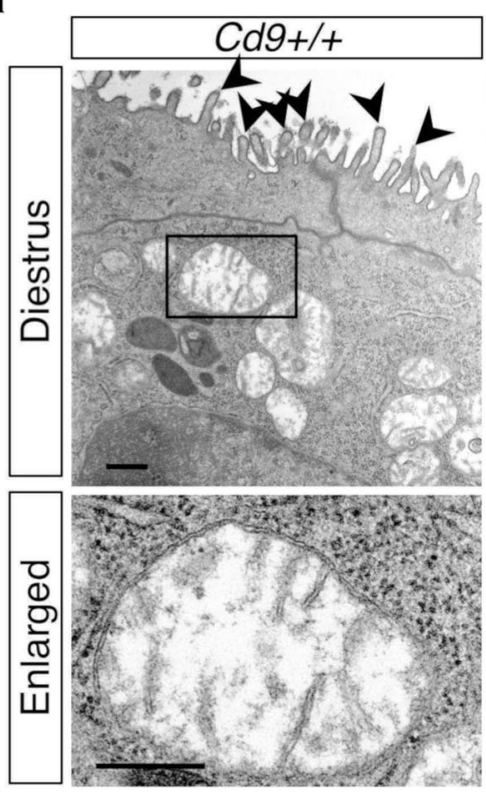

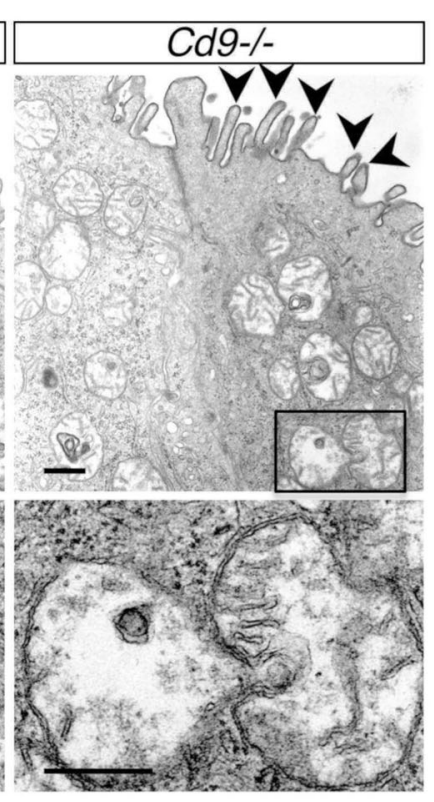

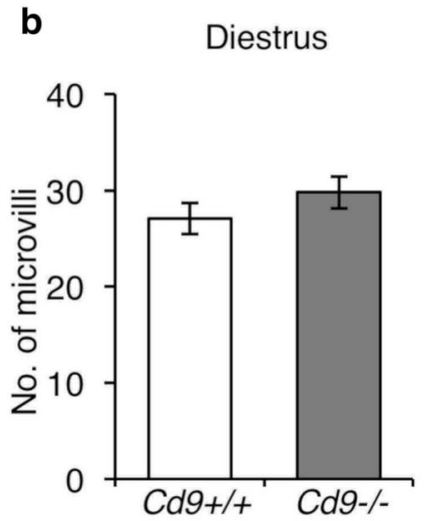

C
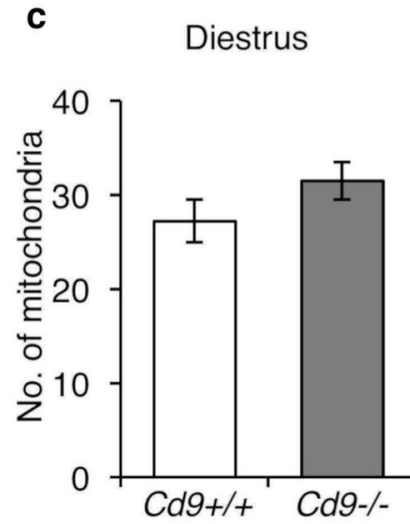

e

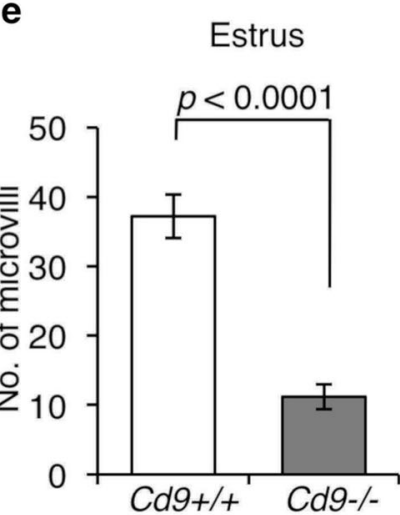

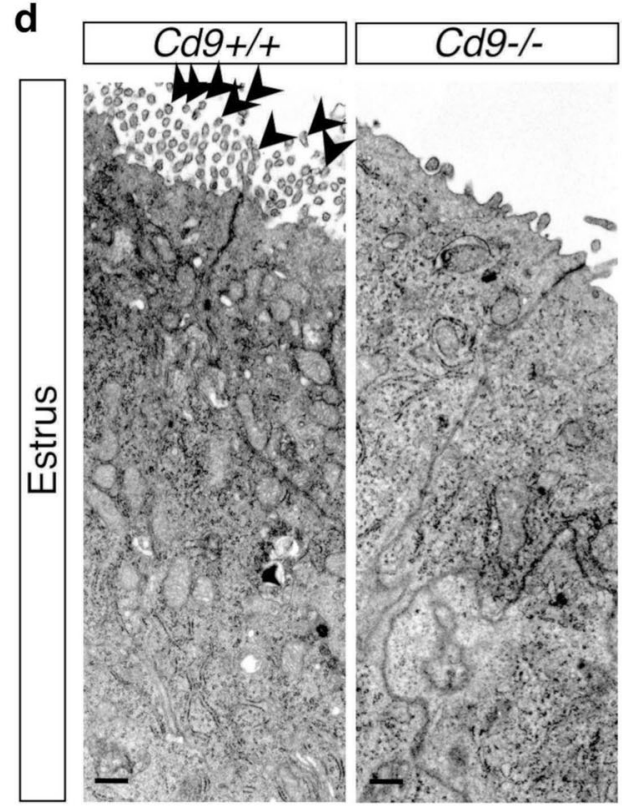

f

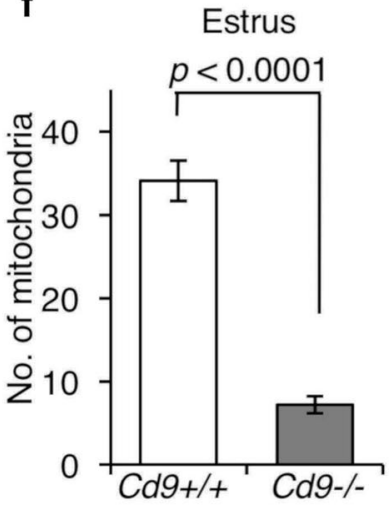

Fig. 4 Ultrastructural features of uterine epithelial cells lacking CD9. a Epithelial cells staged at diestrus in $C d 9^{+/+}$and $C d 9^{-/} T G$ mice. b, c Number of microvilli (arrowheads) and mitochondria at the diestrus stage; values are expressed as mean \pm SE. d Epithelial cells

staged at estrus in $C d 9^{+/+}$and $C d 9^{-/} T G$ mice. e, f Number of microvilli (arrowheads) and mitochondria at the estrus stage; values are expressed as mean $\pm \mathrm{SE}$; scale bars, $500 \mathrm{~nm}$ 
but their fecundity is reduced from their second pregnancy onward because of the failure of uterine remodeling [14]. As a control, we first observed uterine epithelial cells staged at diestrus (Fig. 4a). Regardless of the presence or absence of CD9 proteins, microvilli were formed on the plasma membrane of the epithelial cells in both $C d 9^{+/+}$and $C d 9^{-/-}$ TG mice (Fig. 4a). The number of microvilli was comparable between $C d 9^{+/+}$and $C d 9^{-/-} \mathrm{TG}$ mice $(27.1 \pm 1.6$ and $29.8 \pm 1.7$, respectively; $n=10$ ) (Fig. 4b). Moreover, mitochondria appeared to be immature due to structurally fragile cristae, but the number of mitochondria was indistinguishable between $C d 9^{+/+}$and $C d 9^{-/}$TG mice $(27.2 \pm$ 2.3 and $31.5 \pm 2.0$, respectively; $n=10$ ) (Fig. 4c).

In contrast, at the estrus stage, an enormous number of microvilli were formed in the epithelial cells of $C d 9^{+/+}$ mice, whereas microvilli were rarely formed and shorter in the epithelial cells of $C d 9^{-/}$TG mice $(37.2 \pm 3.1$ vs. $11.2 \pm$ 1.8 , respectively; $n=10 ; p<0.0001$ ) (Figs. $4 \mathrm{~d}$, e). We focused further on the mitochondria, which seemed to mature in the epithelial cells of both groups of mice; but the number of mitochondria was strikingly reduced in $C d 9^{-/} \mathrm{TG}$ mice compared with $C d 9^{+/+}$mice $(7.2 \pm 1.0$ vs. $34.1 \pm 2.4$, respectively; $n=10 ; p<0.0001$ ) (Figs. 4d, f). This result implies that CD9 largely affects membrane organization and cellular homeostasis processes, such as mitochondrial proliferation via microexosome formation (Fig. 5).

\section{Discussion}

In the present study, we demonstrated that CD9 proteins relocate according to uterine cyclical function and are released to the uterine cavity as a component of microexosomes in a uterine-cycle-specific manner. We further showed that CD9 deficiency affects membrane organization and mitochondrial quantity. Our results indicate that, despite its role as an integral membrane protein, CD9 changes its localization according to cellular activity. As CD9 is expressed in a wide variety of cell types, we presume that this phenomenon also occurs in other tissues.

Cytoplasmic proteins relocate in response to external stress [29]. In contrast, membrane-spanning proteins are static and rarely change their location within the membrane. Junctional adhesion molecule A (JAM-A) is a member of the immunoglobulin superfamily and has a single transmembrane domain [30]. It is involved in endothelial cell-leukocyte interactions, contributing to the ring-like structure that forms on the endothelial cell surrounding a transmigrating leukocyte [31]. Unlike other tight junction and adherens junction components, JAM-A accumulates on the plasma membrane at the point of transmigration [32]. Furthermore, the relocalization of plasma membrane proteins is critical for altering cellular polarity and initiating cellular signaling [33]. As shown in Figs. 1b-d, CD9 relocates from the basal region to the apical region during
Fig. 5 Schematic model of CD9 relocalization and release from uterine epithelial cells: green particles indicate microexosomes; CD9 relocates from the basal region to the apical region and the polarity of uterine epithelial cell shifts; in $C d 9^{-/}$epithelial cells, microvilli were rarely formed and shorter; the number of mitochondria was reduced in $C d 9^{-/-} T G$ mice

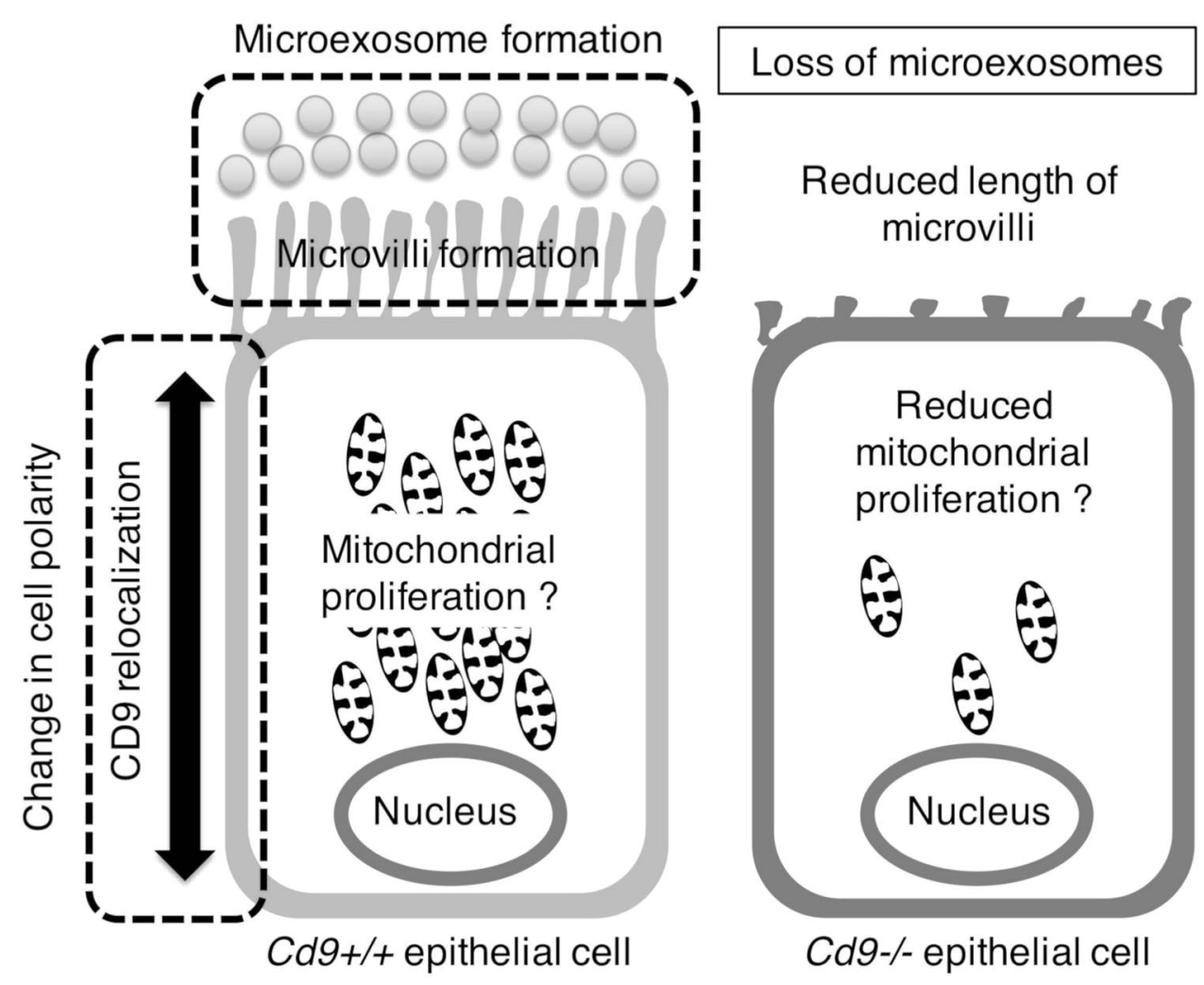


implantation, implying that the cell polarity of uterine epithelial cells shifts. Otherwise, embryos implant on the uterine epithelium of $C d 9^{-/} \mathrm{TG}$ female mice [14]. Therefore, cell polarity may be unaltered in the absence of CD9 proteins, because $C d 9^{--} \mathrm{mESCs}$ succeed in differentiating into three germ layers [34].

Mitochondria change morphologically and proliferate by their fission and fusion [35]. Mitochondrial proliferation also depends on external factors and appears to play a role in the regulation of cell proliferation. In utero, periodic morphological changes are also predicted to demand a massive supply of energy from mitochondria; however, the involvement of mitochondria in uterine function remains less understood.

Exosomes differ structurally from microexosomes [18]. The differences mainly stem from the fact that lipid bilayers are typically formed in exosomes but not in microexosomes. Concordantly, their formation processes are expected to differ. In general, exosomes are formed as a consequence of the fusion of multivesicular late endosomes with the plasma membrane [36]. The multivesicular bodies then fuse to the plasma membrane and release the membrane vesicles as exosomes into the external environment. On the other hand, microexosomes are predicted to be directly released from the plasma membrane without an endosomal pathway. First, selected membrane components targeted in a procedure, including lipids, are extracted from the plasma membrane, presumably by lipid bilayer deformation [16, 18]. In turn, these components are released into the external environment, and concurrently, microvilli are formed on the plasma membrane. CD9 and other tetraspanins are predicted to play a role in the process of lipid bilayer deformation. A clear understanding of the characteristics and functions of exosomes and microexosomes as extracellular structures would greatly advance our attempts to elucidate the molecular mechanisms of phenomena related to intercellular transportation and membrane remodeling.

Acknowledgements This study was supported by a grant from the Ministry of Health, Labor, and Welfare, and a Grant-in-Aid for Scientific Research from the Ministry of Education, Culture, Sports, and Technology of Japan. This work was supported in part by JSPS KAKENHI grant numbers JP16H02094, JP18K16824, and JP16KK0192.

\section{Compliance with ethical standards}

Conflict of interest The authors declare that they have no conflict of interest.

\section{References}

1. Evans J, Salamonsen LA, Winship A, et al. Fertile ground: human endometrial programming and lessons in health and disease. Nat Rev Endocrinol. 2016;12:654-67.
2. Wood GA, Fata JE, Watson KL, et al. Circulating hormones and estrous stage predict cellular and stromal remodeling in murine uterus. Reproduction. 2007;133:1035-44.

3. Tabiasco J, Rabot M, Aguerre-Girr M, et al. Human decidual NKcells: unique phenotype and functional properties -- a review. Placenta. 2006;27(Suppl A):S34-9.

4. Byers SL, Wiles MV, Dunn SL, et al. Mouse estrous cycle identification tool and images. PLoS ONE. 2012;7:e35538.

5. Giudice LC. Growth factors and growth modulators in human uterine endometrium: their potential relevance to reproductive medicine. Fertil Steril. 1994;61:1-17.

6. Orsi NM, Tribe RM. Cytokine networks and the regulation of uterine function in pregnancy and parturition. J Neuroendocrinol. 2008;20:462-9.

7. Mulder EJ, Robles de Medina PG, Huizink AC, et al. Prenatal maternal stress: effects on pregnancy and the (unborn) child. Early Hum Dev. 2002;70:3-14.

8. Jauniaux E, Hempstock J, Greenwold N, et al. Trophoblastic oxidative stress in relation to temporal and regional differences in maternal placental blood flow in normal and abnormal early pregnancies. Am J Pathol. 2003;162:115-25.

9. Gray CA, Bartol FF, Tarleton BJ, et al. Developmental biology of uterine glands. Biol Reprod. 2001;65:1311-23.

10. Fata JE, Ho AT, Leco KJ, et al. Cellular turnover and extracellular matrix remodeling in female reproductive tissues: functions of metalloproteinases and their inhibitors. Cell Mol Life Sci. 2000;57:77-95.

11. Yanez-Mo M, Barreiro O, Gordon-Alonso M, et al. Tetraspaninenriched microdomains: a functional unit in cell plasma membranes. Trends Cell Biol. 2009;19:434-46.

12. Hemler ME. Tetraspanin functions and associated microdomains. Nat Rev Mol Cell Biol. 2005;6:801-11.

13. Park KR, Inoue T, Ueda M, et al. CD9 is expressed on human endometrial epithelial cells in association with integrins alpha(6), alpha(3) and beta(1). Mol Hum Reprod. 2000;6:252-7.

14. Kawano N, Miyado K, Yoshii N, et al. Absence of CD9 reduces endometrial VEGF secretion and impairs uterine repair after parturition. Sci Rep. 2014;4:4701.

15. Raposo G, Stoorvogel W. Extracellular vesicles: exosomes, microvesicles, and friends. J Cell Biol. 2013;200:373-83.

16. Miyado K, Yoshida K, Yamagata K, et al. The fusing ability of sperm is bestowed by CD9-containing vesicles released from eggs in mice. Proc Natl Acad Sci USA. 2008;105:12921-6.

17. Ohnami N, Nakamura A, Miyado M, et al. CD81 and CD9 work independently as extracellular components upon fusion of sperm and oocyte. Biol Open. 2012;1:640-7.

18. Miyado K, Kang W, Yamatoya K, et al. Exosomes versus microexosomes: shared components but distinct functions. J Plant Res. 2017;130:479-83.

19. Cui CH, Uyama T, Miyado K, et al. Menstrual blood-derived cells confer human dystrophin expression in the murine model of Duchenne muscular dystrophy via cell fusion and myogenic transdifferentiation. Mol Biol Cell. 2007;18:1586-94.

20. Takahashi K, Tanabe K, Ohnuki M, et al. Induction of pluripotent stem cells from adult human fibroblasts by defined factors. Cell. 2007;131:861-72.

21. Nishino K, Toyoda M, Yamazaki-Inoue M, et al. Defining hypomethylated regions of stem cell-specific promoters in human iPS cells derived from extra-embryonic amnions and lung fibroblasts. PLoS ONE. 2010;5:e13017.

22. Miyado K, Yamada G, Yamada S, et al. Requirement of CD9 on the egg plasma membrane for fertilization. Science. 2000;287:321-4.

23. Okumura N, Akutsu H, Sugawara T, et al. Beta-catenin functions pleiotropically in differentiation and tumorigenesis in mouse embryo-derived stem cells. PLoS ONE. 2013;8:e63265. 
24. Supangat S, An YJ, Sun Y, et al. Purification, crystallization and preliminary crystallographic analysis of a multiple cofactordependent DNA ligase from Sulfophobococcus zilligii. Acta Crystallogr Sect F Struct Biol Cryst Commun. 2010;66(Pt 12):1583-5.

25. Toshimori K, Saxena DK, Tanii I, et al. An MN9 antigenic molecule, equatorin, is required for successful sperm-oocyte fusion in mice. Biol Reprod. 1998;59:22-9.

26. Ferrara N, Gerber HP, LeCouter J. The biology of VEGF and its receptors. Nat Med. 2003;9:669-76.

27. Guo D, Wang Q, Li C, et al. VEGF stimulated the angiogenesis by promoting the mitochondrial functions. Oncotarget. 2017;8:77020-27.

28. Marx SO, Jayaraman T, Go LO, et al. Rapamycin-FKBP inhibits cell cycle regulators of proliferation in vascular smooth muscle cells. Circ Res. 1995;76:412-7.

29. Damaghi M, Gillies RJ. Lysosomal protein relocation as an adaptation mechanism to extracellular acidosis. Cell Cycle. 2016;15:1659-60.

30. Stamatovic SM, Sladojevic N, Keep RF, et al. Relocalization of junctional adhesion molecule A during inflammatory stimulation of brain endothelial cells. Mol Cell Biol. 2012;32:3414-27.
31. Mamdouh Z, Mikhailov A, Muller WA. Transcellular migration of leukocytes is mediated by the endothelial lateral border recycling compartment. J Exp Med. 2009;206: 2795-808.

32. Weber C, Fraemohs L, Dejana E. The role of junctional adhesion molecules in vascular inflammation. Nat Rev Immunol. 2007;7:467-77.

33. Li S, Esterberg R, Lachance V, et al. Rack1 is required for Vang12 membrane localization and planar cell polarity signaling while attenuating canonical Wnt activity. Proc Natl Acad Sci USA. 2011;108:2264-9.

34. Akutsu H, Miura T, Machida M, et al. Maintenance of pluripotency and self-renewal ability of mouse embryonic stem cells in the absence of tetraspanin CD9. Differentiation. 2009;78:137-42.

35. Ishihara $\mathrm{T}$, Kohno $\mathrm{H}$, Ishihara N. Physiological roles of mitochondrial fission in cultured cells and mouse development. Ann N Y Acad Sci. 2015;1350:77-81.

36. Desrochers LM, Antonyak MA, Cerione RA. Extracellular vesicles: satellites of information transfer in cancer and stem cell biology. Dev Cell. 2016;37:301-9. 